\title{
Ketosis and associated Oxidative Stress in Ruminants
}

\section{Wael M. El-Deeb*}

Department of clinical studies, College of Veterinary Medicine and animal Resources, King Faisal University, Saudi Arabia

Ketosis is a main production disease instigated by negative energy balance, manifested by comparatively elevated concentration of ketone bodies through a simultaneous reduces of blood glucose levels. An impressive elevation in energy necessities during both first stages of lactation and few weeks after parturition makes dairy cattle extremely vulnerable to the state of negative energy balance.

The adaptation of lactating cows to negative energy balance necessitates connections between energy produced from metabolism and its malfunction can happen in many tissues like the adipose tissue and hepatic tissues.

The oxidative stress originated from oxidative process is usually demarcated as an inequity between antioxidants and free radicals at the individual or cellular level. The injury originated from Oxidative process, reactive nitrogen \& oxygen molecules [RNS, ROS] show a protagonist in the beginning or development of plentiful syndromes, like cancer. Free radicals are molecules, which has an additional electron all by itself. Atoms are mainly steady in the ground state. Radical molecule is every molecule with one or more uncoupled electron in the outer membrane, and is talented with independent existence. Oxidative injury is a unique consequence resulted from such inequity and includes radical alteration of cellular macromolecules, cell death caused by necrosis, \& structural tissue destruction.

It was defined that antioxidants is every imperative constituent that impediment, prevent oxidative injury to the target cell or organ. We can say that antioxidants pedal the level of reactive species rather than get rid of them. The aerobic organisms possess a multi-leveled ROS protection system of non-enzymatic and enzymatic antioxidants. Enzymatic antioxidants act as primary defense whereas non-enzymatic antioxidants act as secondary defense against ROS.

Augmented production of radical molecules and establishment of oxidative damage happens as a consequence of increased oxidation of non-esterified fatty acids (NEFA) in liver. Therefore, metabolic profiles as well as anti-oxidative/pro-oxidative situation are a practical device for observing the health status of the animals and reproduction efficacy through the periparturient period.

Cows through the post-partum stage are disputed as energy requirements exceed energy intake, and animals pierce in status of negative energy balance. This activated primarily catabolic trails, which, at the cellular level, augment the creation of radicals' molecules. A strong evidence is stated that oxidative injury is influenced by body condition scoring at parturition and by quantity of milk production and ration constituent (quantity and quality).

There is inverse affiliation between the score of body condition before parturition and the values of oxidative stress (body condition score 4 is much challenged with superfluous oxidative stress than body condition score 2). From another point of view, there is a positive correlation between milk production and oxidative stress (cows with high milk production is usually challenged with extra stress than low producing cows)

Assessment of the oxidant-antioxidant balance by estimating the paraoxonase- 1 activity and malondialdehyde (MDA) values was investigated in cows through the periparturient period.

Research efforts were carried in water buffalo, in Egypt to augment the production curve by enhancement some elements hindering the reproductive efficacy.

Ketosis remains the chief production disorder that decline the production curve in animals. Research investigations were carried on the clinical profile and managemental concern of ketosis in water buffaloes. Glucose and insulin in serum was engaged in buffaloes suffered from ketosis.

Oxidative stress markers have a great role in ketotic water buffaloes. The research results revealed a major upsurge in the values of MDA, nitric oxide in ketotic buffaloes compared to control healthy ones.

Harmful radical molecules are constantly generated through the aerobic metabolism and values can be elevated intensely through amplified creation of necessities or as a consequence of particular disease disorders. The oxidative stress biomarkers were elevated in cattle with subclinical ketosis and those with hyper ketonemia when matched with regular ketonemic people suffered type-1 diabetes mellitus. Definitely healthy cells are capable to compete with radical molecules by the assistance of antioxidant enzyme system. So the oxidant-antioxidant balance should be monitored in ruminants particularly in the periparturient period to prevent ketosis and its associated complications.
*Corresponding author: El-Deeb WM, Department of clinical studies, College of Veterinary Medicine and animal Resources, King Faisal University, Saudi Arabia, Tel: 00966-050-9296154; Fax: 00966-03-5816635; E-mail: drwaeleldeeb@yahoo.com

Received December 19, 2014; Accepted December 20, 2014; Published December 22, 2014

Citation: El-Deeb WM (2015) Ketosis and associated Oxidative Stress in Ruminants. J Veterinar Sci Technol 6: e114. doi:10.4172/2157-7579.1000e114

Copyright: @ 2015 El-Deeb WM. This is an open-access article distributed under the terms of the Creative Commons Attribution License, which permits unrestricted use, distribution, and reproduction in any medium, provided the original author and source are credited. 